\title{
TAGUNG
}

\section{Grenzen der Integration im deutschen und europäischen Recht}

\author{
Frédéric Krumbein und Sebastian Zeitzmann*
}

Wie eng kann die ,ever closer union“"werden? Wo liegen die nationalen rechtlichen Grenzen einer immer engeren Europäischen Union? Dies waren laut Thomas Giegerich die Leitfragen der Tagung. Es gebe einige Bereiche, die nicht supranationalisiert werden könnten. In Deutschland seien dies unter anderem zentrale Budgetentscheidungen, das Gewaltmonopol des Staates bei der Polizei und beim Militär sowie die Sicherung des sozialstaatlichen Fundaments.

Seit Winstons Churchills Aufruf zur Gründung der Vereinigten Staaten von Europa aus dem Jahr 1946 rücke Europa immer enger zusammen, insbesondere durch die Europäische Union. Die Finanz- und Schuldenkrise sei auch eine Krise der Europäischen Union, selbst wenn diese die Krise nicht verursacht habe. Aber es gebe ebenfalls einen positiven Aspekt in der Krise: Dies sei die europaweite öffentliche Debatte über die Zukunft Europas.

Eine Konsequenz aus den unterschiedlichen nationalen Grenzen einer tieferen europäischen Integration könnte in der Notwendigkeit einer differenzierten Integration und einem Europa der verschiedenen Geschwindigkeiten bestehen.

Karl-Peter Sommermann referierte über das Lissabon-Urteil des Bundesverfassungsgerichts (BVerfG) und dessen Auswirkungen. Das Urteil habe unter anderem zu einer Ausweitung der Kompetenzen des Bundestages und des Bundesrates geführt.

\section{The EU between "an ever closer union" and inalienable policy domains of member states}

Gemeinsame Tagung der Universität des Saarlandes, der Europäischen Akademie Otzenhausen und des Arbeitskreises Europäische Integration e.V.

Mit freundlicher Unterstützung der Europäischen Kommission, der Deutschen Forschungsgemeinschaft (DFG), der ASKO-Europa Stiftung, der Europäischen Akademie Otzenhausen, der Hermann und Dr. Charlotte Deutsch Stiftung sowie der Vereinigung der Freunde der Universität des Saarlandes e.V.

21.-23. März 2013, Europäische Akademie Otzenhausen

\section{Introduction}

Introductory remarks: National "red lines" toward European "encroachments"

Thomas GIEGERICH, Europa-Institut, Saarland University, Saarbrücken

The theory of "essential national policies" in the Lisbon Judgment of the German Federal Constitutional Court

Karl-Peter SOMMERMANN, University of Speyer

Comment: Does EU law recognize legal limits to integration?

Leonard BESSELINK, University of Amsterdam

Interaction between EU law and national constitutional law

The EU's common values and the constitutional identities of member states

Christine KADDOUS, University of Geneva

* Dr. Frédéric Krumbein, Geschäftsführer des Arbeitskreises Europäische Integration e.V., Berlin. Sebastian Zeitzmann, Wissenschaftlicher Mitarbeiter am Lehrstuhl von Prof. Thomas Giegerich, Universität des Saarlandes, Saarbrücken.

Der Tagungsband wird als Band 80 der Schriftenreihe des Arbeitskreises Europäische Integration 2014 bei Nomos erscheinen. 
Ein Charakteristikum des Grundgesetzes (GG) bestehe in seiner Offenheit für die europäische Integration und dem grundsätzlichen Fehlen jeder Beschränkung weiterer Integrationsschritte in Art. 24 GG. Bei seiner Verabschiedung hätten die Väter und Mütter des GG noch nicht gewusst, wie die europäische Integration aussehen könnte und hätten deshalb viel Spielraum gelassen.

Das BVerfG habe als nationale Grenzen der europäischen Integration dann aber festgelegt, dass die Grundrechte und die Grundstruktur des Staates durch die europäische Integration nicht angetastet werden dürften. Selbst eine Verfassungsänderung könnte nicht dazu führen, dass Deutschland Mitglied eines europäischen Bundesstaates wird. Eine neue Verfassung wäre in einem solchen Fall erforderlich, da die Ewigkeitsklausel in Art. 79 Abs. 3 des GG einer derart fundamentalen Verfassungsänderung entgegenstehe. Das BVerfG diskutiere aber nicht, ob ein europäischer Bundesstaat nicht auch die Staatsprinzipien von Art. 20 GG schützen könnte. Es wäre durchaus möglich, dass eine solche Konstruktion legal sein könnte: Warum sollten die in Art. 20 GG genannten Verfassungsstrukturprinzipien nicht durch die Europäische Union gesichert werden?

Das BVerfG habe seine Befugnisse bei der Kontrolle weiterer Integrationsschritte kontinuierlich ausgebaut. Das Hauptargument gegen eine unbegrenzte europäische Integration bestehe inzwischen in der Sicherung der demokratischen Selbstbestimmung des deutschen Volkes. Die demokratische Selbstbestimmung sei aber kein überzeugendes Argument, da aus ihm nicht folge, welche Politikfelder supranationalisiert werden dürften und welche nicht. Die Identifikation dieser Politikfelder basiere auf klassischen politischen Theorien, insbesondere der klassischen Staatslehre. Dies sei problematisch. Das GG selbst enthalte keine klare Definition der Politikfelder, die nicht supranationalisiert werden dürften.
Comment I: National Citizenship and Union Citizenship

Helle KRUNKE, University of Copenhagen, and Felix SCHULYOK, European Commission, formerly University of Copenhagen

Comment II: Can discrimination on grounds of nationality ever be obligatory?

Anne THIES, University of Reading

\section{Financial Policy}

National constitutional reservations with respect to tax policy

Suzanne KINGSTON, University College, Dublin

National constitutional reservations with respect to budgetary policy

Florian BECKER, University of Kiel

\section{Security Policy}

National constitutional reservations with respect to internal security policy

Torsten STEIN, Europa-Institut, Saarland University, Saarbrücken

National constitutional reservations with respect to external security policy

Stefan KADELBACH, University of Frankfurt am Main

Comment on national constitutional reservations with regard to the transfer of powers to the $E U$ in the area of internal and external security

Ozan TURHAN, Ozyegin University, formerly Yeditepe University Istanbul

\section{Criminal Law and Procedure}

EU and national constitutional constraints with respect to criminal policy and criminal law

Maria KAIAFA-GBANDI, University of Thessaloniki

Comment: The Europeanization of Criminal Law and Procedure

Lyane SAUTNER, University of Linz

\section{Social Policy}

National constitutional reservations with respect to social policy

Stefanie SCHMAHL, University of Würzburg

Comment I: Limits to national social policy set by EU law

Stein EVJU, University of Oslo 
Insgesamt scheine das BVerfG in Bezug auf künftige Integrationsschritte aber relativ flexibel zu sein. Die verschiedenen Integrationsschritte, die aus den Maßnahmen zur Bewältigung der Finanz- und Schuldenkrise resultierten, seien akzeptiert worden, solange die Mitwirkung des Bundestages gesichert sei. Es bestehe vermutlich noch ein großer Spielraum für weitere Schritte der europäischen Integration. Außerdem sei ein Wandel der Interpretation des GG durch das BVerfG möglich.

Leonard Besselink sprach über Grenzen der europäischen Integration, die das europäische Recht selbst vorschreibt. Die europäische Integration habe seit ihrer Gründung auf die Herstellung und Sicherung des Friedens in Europa durch wirtschaftliche und soziale Prosperität gezielt. Bereits durch die Gründung der Montanunion und die dadurch erfolgte Integration der Kohle- und Stahlproduktion hätten die Nationalstaaten ein deutliches Zeichen der Aufgabe nationaler Souveränität gesetzt. Diese Industrien hätten damals den Kernbereich staatlicher Souveränität betroffen, die Fähigkeit, kriegswichtige Ressourcen bereitzustellen.

Ein allgemeines und begrenzendes Prinzip der Unionskompetenzen bestehe darin, dass die Europäische Union nur Kompetenzen in Bereichen habe, die ihr in den Verträgen explizit zugestanden würden.

Der Vertrag von Lissabon habe außerdem explizit festgeschrieben, dass die Vielfalt der nationalen Identitäten durch die Europäische Union respektiert werden müsse. Aber für Besselink ist es nicht eindeutig, ob dies eine Anerkennung nationaler Grenzen einer europäischen Integration durch das Primärrecht darstelle.

\section{Europäische Werte und nationale Gesetze}

Christine Kaddous referierte über die gemeinsamen Werte der Europäischen Union, das
Comment II: The "Social Purpose" of EU Law (Laval Case)

Roger BLANPAIN, CER Leuven

\section{Cultural Policy}

National constitutional reservations with respect to cultural policy

Kerstin ODENDAHL, Walther Schücking Institute, University of Kiel

Comment I: National language policy within the framework of European law

Xabier ARZOZ SANTISTEBAN, University of the Basque Country, Leioa

Comment II: National media policy within the framework of European law

Pál SONNEVEND, University of Budapest

Comment III: National policy toward religious communities within the framework of European law Monica LUGATO, LUMSA University, Rome

\section{Family and Education Policy}

National constitutional reservations with respect to family policy

Anna STĘIEŃ-SPOREK, University of Gdansk

National constitutional reservations with respect to school and education policy

Hans PECHAR, University of Klagenfurt

Comment: School and Education Policy

Sacha GARBEN, European Commission, formerly

London School of Economics, London

Panel-Discussion: How to square the circle between political necessities and constitutional constraints?

Iris CANOR, College of Management, Rishon LeZion

Oliver DIGGELMANN, University of Zurich

Waltraud HAKENBERG, European Court of Justice, Civil Service Tribunal, Luxemburg

Jo LEINEN, Member of the European Parliament, Strasburg/Brussels

Siegfried MAGIERA, University of Speyer

\section{Concluding Remarks}

Thomas GIEGERICH, Europa-Institut, Saarland University, Saarbrücken 
heißt die Werte, die von allen EU-Mitgliedstaaten geteilt werden.

Werte der Europäischen Union seien der Respekt vor der menschlichen Würde, der Freiheit, Gleichheit und Demokratie sowie vor Menschenrechten und Rechtsstaatlichkeit. Weiterhin müsse die Europäische Union nach Art. 4 des Vertrags über die Europäische Union (EUV) die nationale Identität der Mitgliedstaaten respektieren. Die Europäische Union legitimiere sich durch demokratische Prinzipien. ${ }^{1}$ Die Menschenwürde als Wert stamme ursprünglich aus dem deutschen GG, aber habe sich zu einer europäischen Norm entwickelt und finde sich nun ebenfalls in Art. 2 EUV.

Die Europäische Union habe auch die Verpflichtung, ihre gemeinsamen Werte zu fördern. So gehöre es zu den Zielen der Gemeinsamen Außen- und Sicherheitspolitik, diesen auch in den auswärtigen Beziehungen der Union Geltung zu verschaffen. Der Respekt für diese Normen stelle eine Voraussetzung für eine Mitgliedschaft in der Europäischen Union dar. Die politischen Institutionen der Europäischen Union analysierten vor einer Mitgliedschaft eines Beitrittskandidaten, inwieweit dieser die Werte der Union respektiere. Falls ein Mitgliedstaat nach dem Beitritt diese Werte verletze, so könne er vor dem Europäischen Gerichtshof verklagt werden. Die Werte der Europäischen Union seien abstrakt und dienten als leitende Prinzipien für die Interpretation des europäischen Rechts.

Wie weit begrenze die Klausel über die Achtung der nationalen Identität die europäische Integration? Diese Klausel bestehe seit dem Vertrag von Maastricht und stelle ein Gegengewicht zu den (anderen) gemeinsamen Werten der Europäischen Union dar. Die nationale Identität bezeichne unter anderem die Verfassungstraditionen der Mitgliedstaaten. Die Klausel werde so interpretiert, dass sie den nationalen Verfassungsgerichten eine begrenzte
Kompetenz einräume, den Vorrang der Geltung des EU-Rechts in bestimmten Fällen unter Berufung auf nationale Verfassungsprinzipien einzuschränken. Beispielsweise habe der Europäische Gerichtshof es dem Vereinigten Königreich erlaubt, Bürgern aus Staaten des Commonwealth das Europawahlrecht in Großbritannien zu gewähren, obwohl diese keine britischen Bürger und damit auch keine Unionsbürger seien.

Helle Krunke und Felix Schulyok referierten über die Auswirkungen des europäischen Rechts auf die nationalen Gesetze über die Staatsbürgerschaft. Alle Bürger eines Mitgliedstaats der Europäischen Union verfügten zusätzlich über die Unionsbürgerschaft. Der Entzug der nationalen Staatsbürgerschaft führe auch zu einem Entzug dieser Unionsbürgerschaft und damit zum Verlust der nach europäischem Recht garantierten Freiheiten für EU-Bürger.

Die Mitgliedstaaten könnten die Vergabe und den Entzug der Staatsbürgerschaft selbst regeln, aber sie müssten das europäische Recht dabei respektieren. Darüber hinaus gebe es das internationale Recht, welches den Nationalstaaten bestimmte Grenzen beim Entzug der Staatsbürgerschaft setze. Die Vergabe der Staatsbürgerschaft durch Nationalstaaten könne unter europäischem Recht nur dann problematisch sein, wenn ein Mitgliedstaat einer sehr großen Zahl von Personen seine Staatsbürgerschaft gewähre, da dann möglicherweise nicht sichergestellt sei, dass diese Bürger der Europäischen Union gegenüber loyal seien.

Anne Thies diskutierte die Frage, ob Diskriminierungen aufgrund der Staatsangehörigkeit, die das nationale Recht unter Umständen vorschreibe, nach europäischem Recht zulässig sein können. Grundsätzlich seien solche Diskriminierungen nicht erlaubt. Dieses Prinzip finde sich in der Charta der Grundrechte der Europäischen Union (EU-Charta) und an mehreren Stellen der EU-Verträge.

1 Erklärung von Laeken zur Zukunft der Europäischen Union, in: Europäischer Rat: Schlussfolgerungen des Vorsitzes. Europäischer Rat (Laeken) 14. und 15. Dezember 2001, SN 300/1/01, S. 19-26. 
Der Europäische Gerichtshof habe beispielsweise geurteilt, dass in Großbritannien der $\mathrm{Zu}-$ gang zu Universitäten und zu Stipendien für EU-Bürger wie für britische Bürger geregelt werden müsse. Allerdings müssten beispielsweise Ärzte die nationalen Vorgaben erfüllen, um eine Zulassung zu erhalten. Deutsche Bürger genössen im Ausland diplomatischen Schutz durch die Bundesrepublik, und hier stelle sich die Frage, ob EU-Bürger den gleichen Schutz durch deutsche Auslandsvertretungen erhalten könnten. Wenn EU-Bürger in deutschen Auslandsvertretungen nach diplomatischem Schutz fragten, gewähre Deutschland diesen. Aber es stelle sich die Frage, ob Drittstaaten diesen Schutz vollständig anerkennen müssten.

\section{Nationale Grenzen im Steuer- und Haushaltsrecht}

Unterschiedliche Steuerregime waren das Thema des Vortrags von Suzanne Kingston. Die verschiedenen EU-Staaten verfügten über sehr unterschiedliche Steuersätze und -philosophien. Die Europäische Union habe offiziell keine eigene Steuerpolitik, weil sie nicht über die Kompetenz verfüge, im Bereich der direkten Steuern Harmonisierungsmaßnahmen zu erlassen. ${ }^{2}$ Allerdings hätten sich durch die Finanz- und Schuldenkrise wichtige Veränderungen in diesem Bereich ergeben. Vor der Krise habe das Thema Steuern auf der Unionsebene keine wichtige Rolle gespielt. Ein Ziel der Europäischen Union sei es aber damals schon gewesen, eine stärkere Harmonisierung bei den national unterschiedlichen Steuersätzen herbeizuführen.

Seit der Krise spiele das Thema eine zunehmend wichtigere Rolle. Verschiedene Maßnahmen der Europäischen Union zielten auf eine Überwindung der Finanz- und Schuldenkrise und nähmen dabei die Einnahmen des Staates mit in den Blick. Durch Entscheidungen des Europäischen Gerichtshofes und durch die Eurokrise sei die Steuergesetzgebung in- zwischen keine ausschließliche Kompetenz der Mitgliedstaaten mehr.

Thomas Giegerich trug ein Manuskript von Florian Becker vor, der selbst nicht an der Tagung teilnehmen konnte. Der Vortrag behandelte die Frage, inwieweit in Bezug auf die Haushaltspolitik nationale verfassungsrechtliche Grenzen existierten, welche die Europäische Union nicht überschreiten dürfe.

In Deutschland habe das BVerfG die Haushaltssouveränität des Bundestages unterstrichen. Der Bundestag dürfe seine Haushaltssouveränität nie an die Europäische Union abgeben, da sie unter die Ewigkeitsklausel des GG falle. Die grundlegenden Rechte des Parlaments müssten gewahrt bleiben und das Budgetrecht falle unter diese grundlegenden Rechte. Außerdem würde sonst die demokratische Selbstbestimmung des deutschen Volkes verletzt werden. Damit setze das BVerfG einer weiteren Vertiefung der Europäischen Union in budgetärer Hinsicht eine Grenze.

Die Übernahme umfangreicher Garantien für die Schulden ausländischer Staaten sei aber noch durch den Entscheidungsspielraum des Parlaments gedeckt, solange der Bundestag seine Budgethoheit als solche behalte. Eine Verfassungswidrigkeit von Haushaltsbeschlüssen des Bundestages sei aber unwahrscheinlich, weil dieser dafür offensichtlich untragbare Risiken für den Haushalt eingehen müsse. Da die Ökonomen aber in der Regel keine einheitliche Meinung über das Risiko einer Budgetentscheidung verträten, könne eine Entscheidung des Bundestages in Haushaltsfragen kaum verfassungswidrig sein. Zentral bleibe vielmehr, dass dem Bundestag jede haushaltsrelevante Entscheidung vorbehalten bleiben müsse. Darüber hinaus müssten der Bundestag und der Bundesrat über alle budgetrelevanten Sachverhalte durch die Bundesregierung und die Europäische Union zeitgerecht informiert werden.

2 Vgl. Art. 113 Vertrag über die Arbeitsweise der Europäischen Union (AEUV). 


\section{Grenzen in der inneren und äußeren Sicherheit}

Torsten Steins Vortrag behandelte nationale Grenzen der europäischen Integration im Bereich der inneren Sicherheit. Die innere Sicherheit falle im Allgemeinen in den ausschließlichen Zuständigkeitsbereich der Mitgliedstaaten. Die Europäische Union verfüge aber über die Möglichkeit, die Zusammenarbeit in der inneren Sicherheit zu fördern, zum Beispiel die Zusammenarbeit der nationalen Polizeibehörden.

Im Lissabon-Urteil des BVerfG tauche der Bereich der inneren Sicherheit nicht auf. Dafür werde das Strafrecht erwähnt. Der ,harte“ Bereich der inneren Sicherheit, insbesondere das Gewaltmonopol der Polizei, sei im LissabonUrteil nicht angesprochen worden. Aber jeder Versuch der Europäischen Union, in diesem Bereich Kompetenzen an sich zu ziehen, könne vergleichsweise einfach durch den Vorrang der nationalen Souveränität abgewehrt werden.

Stefan Kadelbach widmete sich der äußeren Sicherheitsdimension und möglichen nationalen Grenzen einer europäischen Integration in diesem Politikfeld. Das BVerfG habe geurteilt, dass es im Grundgesetz nur zwei Fälle des legitimen Einsatzes von militärischer Gewalt gebe: bei der nationalen Selbstverteidigung und innerhalb eines Systems kollektiver Sicherheit. ${ }^{3}$ Das bedeute, dass im Prinzip keine europäische Militärmission, die nicht durch die Vereinten Nationen oder die $\mathrm{NATO}^{4}$ unterstützt werde, verfassungsrechtlich legitim sein könne. Die Gemeinsame Außen- und Sicherheitspolitik werde durch das BVerfG nicht als ein System kollektiver Sicherheit angesehen.

Hinzu komme, dass jeder Einsatz des Militärs vorab durch den Bundestag genehmigt werden müsse. Diese Vorbehalte dürften auch nicht supranationalisiert, das heißt an die europäische Ebene abgegeben werden. Andere Staaten würden ähnliche Vorbehalte kennen, aber kein
Staat definiere den Bereich der äußeren Sicherheit als einen essenziellen Politikbereich souveräner Nationalstaaten.

Ozan Turhan referierte über türkische Vorbehalte gegen eine Supranationalisierung der inneren und äußeren Sicherheit bei einer EUMitgliedschaft. Die türkische Teilnahme an der Kooperation im Bereich der inneren und äußeren Sicherheit sei aufgrund der geostrategisch wichtigen Lage der Türkei von großer Bedeutung. Die türkische Verfassung verbiete in Art. 6 die Übertragung von Souveränität auf supranationale oder internationale Organisationen derzeit vollständig. Aufgrund des türkischen Beitrittswunsches zur Europäischen Union werde dieser Artikel viel diskutiert.

Internationale Menschenrechtsverträge stünden in der nationalen Normenhierarchie allerdings höher als die türkische Verfassung und sonstiges türkisches Recht. Internationale Verträge, die keine Menschenrechte enthielten, stünden aber nicht über der Verfassung der Türkei. Insgesamt bestehe im türkischen Recht keine Klarheit über den Primat der türkischen Verfassung oder des internationalen Rechts. Es gebe in dieser Hinsicht widerstreitende Auslegungen sowie keine einheitliche Rechtsprechung und Rechtsetzung.

\section{Das Strafrecht nach dem Vertrag von Lissabon}

Das Strafrecht ist nach Maria Kaiafa-Gbandi das schärfste Instrument, das Staaten nutzen, um ihre Gesellschaften zu kontrollieren. Aus diesem Grund sei das Strafrecht eng mit Menschenrechten verknüpft, um staatlichen Missbrauch zu verhindern. Seit dem Vertrag von Lissabon biete das EU-Primärrecht einerseits einen besseren Schutz der Grundrechte der EU-Bürger und andererseits neue Möglichkeiten der europäischen Kooperation im Bereich des Strafrechts.

Bereiche mit EU-Kompetenzen fänden sich unter anderem in der Domäne von schweren

3 Art. 87a Abs. 2 und Art. 24 Abs. 2 GG.

4 North Atlantic Treaty Organization. 
Verbrechen. ${ }^{5}$ Die Europäische Union könne ebenfalls das Strafrecht dafür nutzen, um ihre Politiken durchzusetzen, in denen sie über eine ausschließliche Kompetenz verfüge. ${ }^{6}$ Diese Klausel sei aber zu weit gefasst, selbst wenn das Strafrecht nur als letztes Mittel zur Durchsetzung der EU-Politiken eingesetzt werden dürfe.

Das ,Ultima-Ratio'-Prinzip stelle das wichtigste Prinzip im Strafrecht dar und sei eng verwandt mit dem Prinzip der Verhältnismäßigkeit. Das ,Ultima-Ratio'-Prinzip bedeute, dass die Anwendung des Strafrechts nur als letztes Mittel erlaubt sei, das heißt wenn keine anderen Mittel zum gleichen Ergebnis führen würden. Diese beiden Prinzipien würden in der Europäischen Union nicht ausreichend respektiert. Beispielsweise kriminalisiere der EUAntiterrorismus-Rahmenbeschluss aus dem Jahr 2008 den Kampf gegen den Terrorismus einseitig. ${ }^{7}$ Ein weiteres zentrales Prinzip sei das ,Lex-Certa'-Prinzip, welches klare rechtliche Formulierungen von Strafgesetzen vorschreibe: Die Bürger müssten immer wissen, welche Handlungen kriminell seien und welche nicht.

Positiv sei zu erwähnen, dass die Europäische Union grundsätzlich das Prinzip respektiere, dass ein Mitgliedstaat selbst entscheiden könne, ob er für eine Gesetzesübertretung strafrechtliche Maßnahmen ergreifen möchte oder nicht.

Lyane Sautner diskutierte die Europäisierung des Strafrechts. Das Strafrecht sei fest in den nationalen Traditionen verankert und lasse sich deshalb nicht leicht europäisieren. Ein erster Bereich der Europäisierung betreffe schwere Verbrechen. Terrorismus und Menschenhandel könnten als schwere Verbrechen bezeichnet werden, aber die beste Methode, um diese Verbrechen zu bekämpfen, sei nicht das Straf- recht. Zu oft verwende die Europäische Union strafrechtliche Maßnahmen als Symbolpolitik.

Eine Harmonisierung des Strafrechts erfordere ein hohes Maß an Vertrauen in die Strafsysteme der europäischen Mitgliedstaaten, da es kaum gemeinsame Standards gebe. Diese gemeinsamen Standards müssten stärker entwickelt werden. Ein positives Beispiel für die Entwicklung gemeinsamer Standards finde sich in einer europäischen Richtlinie zum Schutz der Opfer von Verbrechen.

\section{Die Sozialpolitik der Europäischen Union}

Stefanie Schmahl eröffnete ihre Präsentation mit der These, dass die Europäische Union aufgrund von Kompetenzgewinnen mittlerweile in einzelnen Bereichen mit einem Sozialstaat vergleichbar sei. Mehrere Verfassungsgerichte von EU-Mitgliedstaaten hätten dies bestätigt, darunter das französische und das polnische, allerdings nationale Vorbehalte geltend gemacht. Vor allem das BVerfG habe die Relevanz sozialer Prinzipien in der Europäischen Union betont.

Im Primärrecht sei die Sozialpolitik bereits seit den Römischen Verträgen angelegt, unter anderem im heutigen Art. 157 AEUV. Seitdem habe ihre Bedeutung stetig zugenommen, wie sich an der Formulierung ,wettbewerbsfähige soziale Marktwirtschaft" in Art. 3 Abs. 3 EUV zeige. Die Sozialpolitik leite sich in erster Linie aus den Grundfreiheiten und der Unionsbürgerschaft ab und sei in hohem Maße durch die Rechtsprechung des Europäischen Gerichtshofs determiniert. Diese habe sich vor allem von der Unterscheidung zwischen wirtschaftlich und nicht wirtschaftlich tätigen Personen wegbewegt, was die Gewährleistung sozialer Leistungen angehe. Sekundärrechtlich werde die Gewährleistung solcher Leistungen in erster Linie aus der Arbeitnehmerfreizügigkeit

5 Art. 83 AEUV.

6 Art. 83 Abs. 2 AEUV

7 Rahmenbeschluss 2008/919/JI des Rates vom 28. November 2008 zur Änderung des Rahmenbeschlusses 2002/475/JI zur Terrorismusbekämpfung, in: Amtsblatt der EU, Nr. L 330 vom 9. Dezember 2008, S. 21-23. 
abgeleitet und stehe in einem Gegenseitigkeitsverhältnis zu wirtschaftlicher Aktivität.

Der EuGH fordere von Mitgliedstaaten für Beschränkungen sozialer Leistungen in Bezug auf grenzüberschreitende Sachverhalte eine objektive Rechtfertigung. In seiner Rechtsprechung, vor allem den Urteilen ,Martínez Sa$1 a^{68}$, Vidal ${ }^{69}$ und ,Förster ${ }^{610}$ betone er, dass der Grundsatz der Nichtdiskriminierung stets eine soziale Interpretation erfordere. Im Gegensatz dazu stehe der Europäische Gerichtshof für Menschenrechte, welcher in seiner Rechtsprechung lange Zeit finanzielle Eigenleistungen als notwendige Voraussetzung für den eigentumsrechtlichen Schutz von Ansprüchen auf soziale Leistungen nach Art. 1 des Zusatzprotokolls zur Europäischen Menschenrechtskonvention verlangt habe, heute jedoch eine weniger strenge Linie verfolge. Schmahl argumentierte, dass das Erfordernis einer echten Verbindung des Begünstigten zu dem die Leistung gewährenden Staat oder zumindest eines gewissen Niveaus von Integration in dessen Gesellschaft eine angemessene Voraussetzung für die Gewährung von Sozialleistungen sei und es ein ausgewogenes Verhältnis zwischen Individualinteressen und gesellschaftlichen Interessen zu gewährleisten vermöge.

In ihrer Schlussfolgerung hinterfragte Schmahl, ob man tatsächlich von einer echten EU-Sozialpolitik sprechen könne. Sie verneinte dies und stellte fest, dass eine Verdrängung nationaler Sozialstaatlichkeit durch die Sozialpolitik der Union nicht wünschenswert sei. Diese stelle jedoch eine wichtige Ergänzung zu dem auf mitgliedstaatlicher Ebene gewährleisteten Schutz dar.

Stein Evju betrachtete die Thematik aus der Drittstaatsperspektive Norwegens als einem Mitglied des Europäischen Wirtschaftsraumes (EWR). Er hob hervor, dass für Norwegen die Europäische Union nur eine Säule des EWR darstelle. Zudem existiere in den skandinavischen Staaten keine formale Verfassungsgerichtsbarkeit.

Norwegen sei ein wachsender Arbeitsmarkt mit etwa 200.000 Arbeitsimmigranten, von denen 50 Prozent aus den neuen EU-Mitgliedstaaten kämen. 10 Prozent aller Arbeitskräfte seien EU-Bürger. Sie profitierten vom norwegischen Tarifverhandlungssystem, welches als spezifische Ausprägung des ,nordischen Modells" seit über 100 Jahren stabil sei. Es garantiere bei vergleichsweise hohen Steuern einen umfassenden Sozialstaat.

Der Einfluss der EU-Gesetzgebung und Rechtsprechung des EuGH sei in Norwegen zwar weniger stark und anders ausgeprägt als in Schweden oder Dänemark, den nordischen „Schwesterstaaten“ Norwegens. Allerdings verleugnete Evju keineswegs eine gewisse Unruhe, welche vor allem das EuGH-Urteil ,La$\mathrm{val}^{111}$ in Norwegen ausgelöst habe. $\mathrm{Zu}$ groß erschienen die Eingriffe in die nationale Domäne der Sozialpolitik, vor allem in Bezug auf das Arbeits- und Streikrecht. Es sei deutlich, dass der Ansatz des EuGH auf Dauer nicht haltbar sein werde. Andernfalls drohe aufgrund der Diskrepanz zwischen EU- und mitgliedstaatlicher Interessenssphäre eine Gefährdung der Einheit und Beständigkeit der Europäischen Union und des EWR.

Roger Blanpain unterstrich die Bedeutung der Europäischen Union im Bereich der Sozialpolitik, obwohl deren Kernpunkte nicht in den Kompetenzkatalog der Union fielen und Einstimmigkeitserfordernisse im Rat die Entscheidungsfindung bis zur Unmöglichkeit erschwerten. Festgehalten werden könne jedoch, dass die Europäische Union durch die Gewährleistung der Grundfreiheiten Sozialdumping fördere, dieses aber gerade vermieden werden sollte.

8 EuGH, Rs. C-85/96 (Martínez Sala), Slg. 1998, I-2691.

9 EuGH, Rs. C-344/89 (Vidal), Slg. 1991, I-3245.

10 EuGH, Rs. C-158/07 (Förster), Slg. 2008, I-8507.

11 EuGH, Rs. C-341/05 (Laval), Slg. 2007, I-11767. 
Grundsätzlich schloss Blanpain sich Plädoyers für mehr Europa an. Arbeitsrecht und -verhältnisse seien jedoch essenziell nationalstaatliche Materien. „Europa“ unterwandere die nationalen Arbeitsmärkte. Ein Problem sei beispielsweise die gegenseitige Anerkennung von Selbstständigkeitszeugnissen, welche zum Missbrauch von durch die Mitgliedstaaten gewährten sozialstaatlichen Leistungen führen könnte.

Der Bewertung des Urteils ,Laval` durch Evju als falsch vermochte sich Blanpain nicht anzuschließen. Stattdessen begrüßte er das Urteil ausdrücklich, da es geeignet sei, einem gegenwärtigen Mangel an sozialpolitischer Solidarität, sowohl zwischen den Staaten, als auch zwischen Arbeitgebern wie -nehmern entgegenzuwirken. Mehr Solidarität würde letztlich den Arbeitnehmern, vor allem den schwächsten unter ihnen, zugutekommen. Weniger Nationalismus und mehr Integration im Bereich der Sozialpolitik seien daher wünschenswert.

\section{Handeln der EU im Bereich der Kultur}

Kerstin Odendahl begann mit der Frage, ob in der Kulturpolitik ein Kompetenztransfer auf die Union überhaupt erlaubt sei. Das BVerfG habe in seinem Lissabon-Urteil festgehalten, dass Kultur, und damit unter anderem die Sprache, Familien, Schule und Ausbildung, Medienregulierung und Religion, zum unantastbaren Kerngehalt der Identität des GG gehöre. Dieser Ansicht zufolge ist ein entsprechender Kompetenztransfer nicht erlaubt. Verfassungsgerichte anderer Mitgliedstaaten, vor allem Frankreichs und Polens, verträten ähnliche Positionen, würden allerdings auf einen NegativKompetenzkatalog, wie er durch das BVerfG festgelegt worden sei, verzichten. Das tschechische Verfassungsgericht schließe einen solchen sogar explizit aus. Generell lasse sich nur in Deutschland die Kultur explizit innerhalb vergleichbarer Negativ-Kompetenzkataloge finden. Auch betrachte kein anderes europäisches Verfassungsgericht die Kultur in gleicher Weise als relevant für die Demokratie wie das BVerfG.
Odendahl erinnerte daran, dass die EU-Verträge eine „europäische Kultur“ vorsähen. Da es somit eine gemeinsame europäische Kultur gebe, welche der mitgliedstaatlichen Domäne entzogen sei und auf EU-Ebene gefördert und bewahrt werden müsse, sei das Lissabon-Urteil in diesem Punkt nicht vollständig mit dem Primärrecht vereinbar.

$\mathrm{Zu}$ den Elementen der Kultur zählten neben dem anthropologischen und sozialen auch solche des Wandels und der Offenheit. So greife die Europäische Union durch ihre Sekundärrechtsetzung selbst in kulturelle Charakteristika und Besonderheiten der Mitgliedstaaten ein. Als Beispiel wurden harmonisierende Maßnahmen im Bereich des Lebensmittelrechts sowie das Glühbirnenverbot genannt.

Abschließend fragte Odendahl, warum das BVerfG der Kultur wohl eine so starke Rolle zugestehe. Sie argumentierte, dass Deutschland Kultur generell stark fördere, sei es durch die „Kulturhoheit der Länder“ oder als „Kulturstaat". Dieser finde sich so zwar nicht im Grundgesetz, wohl aber in der Rechtsprechung des BVerfG. Allerdings falle es schwer, sich vorzustellen, dass Kultur letztlich nur in Deutschland diesen besonderen Status innehabe.

Xabier Arzoz Santisteban widmete sich im Anschluss der Rolle der Sprachen in der Europäischen Union. Er hielt fest, dass diese nicht nur durch die europäische Integration, sondern allgemein durch die Globalisierung und die Dominanz des Englischen beeinflusst sei. Die verfassungsrechtliche Bedeutung der Sprachen wurde am Beispiel Frankreichs dargelegt. Dort wurde 1992 - parallel zum Vertrag von Maastricht, welcher die Sprachenvielfalt in der Union primärrechtlich kodifizierte - die Bedeutung des Französischen in der Verfassung niedergeschrieben. Dies habe zur Beschränkung anderer Sprachen in der französischen Öffentlichkeit geführt. Auch Polen und ähnlich die Slowakische Republik würden der Sprache einen Stellenwert als konstitutives Element nationaler Identität einräumen. 
Arzoz Santisteban warf im Anschluss die Frage auf, ob das Unionsrecht einen Einfluss dahingehend ausübe, dass es nationale Sprachenpolitik gewissen Einschränkungen unterwerfe. Aus der Rechtsprechung des EuGH, vor allem dem Urteil ,Anita Groener ${ }^{12}$, sei eine Antwort nicht eindeutig möglich. Allerdings könne festgehalten werden, dass EU-Gesetzgebung in Bezug auf nationale Sprachenpolitik mit einer gewissen Vorsicht erfolge. Die Sprachenvielfalt und ihre besondere Anerkennung und Bewahrung durch das EU-Primärrecht wurde abschließend noch einmal betont.

Pál Sonnevend untersuchte im Anschluss die nationale Medienpolitik innerhalb des vom EU-Recht gesetzten Rahmens. Er hielt zunächst fest, dass das Unionsrecht in diesem Bereich sehr zurückhaltend sei. Im Primärrecht würden Medien nur im Protokoll 29 über den öffentlich-rechtlichen Rundfunk in den Mitgliedstaaten ${ }^{13}$ erwähnt, es gebe mit nur einer einschlägigen Richtlinie, der MediendienstRichtlinie, ${ }^{14}$ kaum Sekundärrecht und nur wenig ,soft law' in Form von Empfehlungen.

Sonnevend analysierte hernach das Verhältnis von Art. 2 EUV und Art. 51 Abs. 1 EU-Grundrechtecharta. Dafür zog er vor allem das aktuelle EuGH-Urteil , Åkerberg Fransson' ${ }^{15}$ heran, welches hinsichtlich letzterer Vorschrift eine Bestätigung der bisherigen Rechtsprechung des EuGH darstelle, wonach vom EURecht geregelte Sachverhalte, somit auch Grundwerte, in den Geltungsbereich der europäischen Grundrechte fielen.

In einem nächsten Schritt nahm Sonnevend Bezug auf das neue ungarische Medienrecht von 2010, welches zwar der Audiovisuellen Mediendienst-Richtlinie der Europäischen Union, angeblich aber nicht der EU-Grundrechtecharta unterfalle. Diese Konstellation habe dazu geführt, dass de facto die Kritik der Europäischen Kommission am ungarischen Medienrecht „keine Konsequenzen“ nach sich gezogen habe. Sonnevend schloss daraus, dass Art. 51 Abs. 1 EU-Grundrechtecharta die effektive Durchsetzung der EU-Grundwerte verhindere. Solange die Grundfreiheiten des AEUV nicht berührt seien, bleibe die mitgliedstaatliche Medienpolitik vom Unionsrecht weitgehend unberührt.

Als Lösung dieser Problematik schlug Sonnevend eine extensive Auslegung des Art. 51 Abs. 1 EU-Grundrechtecharta vor.

Monica Lugato referierte abschließend über die Stellung religiöser Organisationen in der Europäischen Union. Eine Kompetenz der Europäischen Union sei in diesem Bereich nicht ersichtlich. Allerdings schreibe Art. 17 AEUV vor, dass in Bezug auf mitgliedstaatliche Kirchen und religiöse Einrichtungen sowie Gemeinschaften Eingriffe durch die Europäische Union nicht gestattet seien. Die Institutionen der Union hätten jedoch dann hinsichtlich der Religionsausübung in den Staaten ein Mitspracherecht, wenn die Grundfreiheiten, vor allem die Dienstleistungsfreiheit berührt würden.

Hervorzuheben sei jedoch die Rolle der Religion hinsichtlich ihres grundrechtlichen Charakters und ihrer Position als EU-Grundwert. So habe der Europäische Gerichtshof eine $\mathrm{Zu}-$ ständigkeit u.a. bei Fragen der religiösen Verfolgung. Als Grundwert sei die Religionsfreiheit vor allem für Beitrittskandidaten relevant, die ohne eine Achtung und eine aktive Förderung aller Grundwerte nicht Mitglied der Union werden könnten.

Abschließend hielt Lugato fest, dass der Bereich der religiösen Organisationen in die mitgliedstaatlichen Kompetenzen fiele, aber mehr

12 EuGH, Rs. C-379/87 (Anita Groener), Slg. 1989, 3967.

13 Amtsblatt der EU, Nr. C 326 vom 26. Oktober 2012, S. 312.

14 Richtlinie 2010/13/EU des Europäischen Parlaments und des Rates vom 10. März 2010 zur Koordinierung bestimmter Rechts- und Verwaltungsvorschriften der Mitgliedstaaten über die Bereitstellung audiovisueller Mediendienste (Richtlinie über audiovisuelle Mediendienste), in: Amtsblatt der EU, Nr. L 95 vom 15. April 2010, S. 1-24.

15 EuGH, Rs. C-617/10 (Åkerberg Fransson), Slg. 2013. 
und mehr einer gerichtlichen Kontrolle durch die Luxemburger und Straßburger Gerichtshöfe unterzögen würde.

\section{Familien- und Bildungspolitik im Unionsrecht}

Der Beitrag von Anna Stepien-Sporek, verlesen von Thomas Giegerich, widmete sich den mitgliedstaatlichen verfassungsrechtlichen Ansätzen hinsichtlich einer gemeinsamen Familienpolitik. Jeder Staat habe zwar seine eigene Familienpolitik, aber die dahinterstehenden Ansätze und Herausforderungen seien vergleichbar. Dennoch sei eine Hinwendung zu einem harmonisierten europäischen Familienrecht nicht erkennbar. Darüber hinaus sei es zweifelhaft, ob eine solche Harmonisierung überhaupt möglich sei.

Zunächst wurde das in der polnischen Verfassung kodifizierte Familienrecht angesprochen, welches sich größtenteils kaum von dem anderer Mitgliedstaaten unterscheide. Grundsätzlich sei es vereinbar sowohl mit den internationalen Verträgen zum Schutz der Menschenrechte als auch mit der EU-Grundrechtecharta. Allerdings seien einige Bereiche reformbedürftig.

Es folgte eine Gegenüberstellung europäischer familienrechtlicher Vorschriften, die es gegenwärtig, vor allem begründet durch den Vertrag von Maastricht, gebe. Ein gewisser Grundbestand an Harmonisierung bestehe, vor allem im Bereich des Ehescheidungsrechts, der elterlichen Verantwortung sowie hinsichtlich von Unterhaltspflichten. Andere Bereiche, zum Beispiel Ehescheidungs- oder Kindesabstammungsrecht, seien bisher von jeder Harmonisierung völlig ausgenommen. Allerdings bestünden teilweise bilaterale Vereinbarungen zwischen den Mitgliedstaaten.

Abschließend wurde festgehalten, dass es für ein einheitliches Unionsfamilienrecht aufgrund religiöser und kultureller Unterschiede zwischen den Staaten zu früh sei. Eine Harmonisierung vor allem in den Bereichen Rechtsprechung und gegenseitige Anerkennung so- wie Durchsetzung von Urteilen wäre hingegen wünschenswert.

Hans Pechar befasste sich im Folgenden mit intraeuropäischer Mobilität von Studierenden, wobei er sich vor allem auf das nicht konfliktfreie Verhältnis zwischen Österreich und Deutschland konzentrierte. Er beschrieb zunächst das besondere offene Zulassungssystem in Österreich, welches unter anderem keinen Numerus clausus kenne, aber ursprünglich mit Restriktionen für Ausländer einhergegangen sei. Der EuGH habe dies 2005 für unionsrechtswidrig erklärt, woraufhin Österreich sein System angepasst und einen Numerus clausus eingeführt, zugleich aber für Medizin eine Quotenregelung geschaffen habe, welche in absoluten Zahlen Österreicher bevorzuge. Dies sei mit der Aufrechterhaltung des Gesundheitssystems begründet worden, ein Disput mit der Europäischen Union bestehe allerdings weiterhin.

Pechar hielt fest, dass die Hochschulausbildung durch nationale Steuern finanziert werde und in ein nicht harmonisiertes Sozialmodell eingebettet sei. Der stillschweigenden Voraussetzung, dass die Studierendenmobilität letztlich zu einem Nullsummenspiel führe, erteilte er eine klare Absage. Eine symmetrische Mobilität zwischen den Staaten bestehe nicht. Vielmehr gebe es große Ungleichgewichte. Vor allem kleine Staaten seien negativ betroffen, dies umso mehr, wenn sie mit größeren Staaten eine Sprache teilten. Die EU-Nichtdiskriminierungspolitik resultiere daher gerade in einer Diskriminierung von Studierenden kleiner Mitgliedstaaten.

Pechar schlug als Lösungsmöglichkeit Kompensationstransfers zwischen den Mitgliedstaaten vor, welche bi- oder multilateraler Natur sein könnten. Anderenfalls drohe die gegenwärtige Belastung zu einer Gefährdung für die Solidarität zwischen den europäischen Bürgern zu werden.

Sacha Garben befasste sich in ihrem Kommentar mit der Schul- und Bildungspolitik. Sie 
bezeichnete die zugrundeliegende primärrechtliche Vorschrift, Art. 165 AEUV, als Herrin über Handlungsbefugnisse und auch die Zurückhaltung bei deren Ausübung. Unter Hinweis darauf, dass dies nicht bedeute, dass Politikbereiche die Europäische Union nicht beträfen, nur weil sie nicht in deren Kompetenzbereiche fielen, zeigte sie die Möglichkeit von „Harmonisierung durch die Hintertür“" auf. Als Beispiel zitierte sie das EU-Tabakwerbeverbot, das auf die allgemeine Harmonisierungskompetenz des Art. 114 AEUV gestützt worden sei. Zugleich machte sie deutlich, dass Harmonisierung gewissermaßen „durch das Badezimmerfenster" beziehungsweise in aller Heimlichkeit erfolgen könne, sei es durch informelle intergouvernementale Zusammenarbeit oder gänzlich außerhalb der Europäischen Union, wie es mit dem Bologna-Prozess geschehen sei.

Diese Optionen stellten für Garben aber einen lediglich unbefriedigenden Status quo dar. Sie sah das Problem in einer zu eingeschränkten Kompetenz durch Art. 165 AEUV, welche zu einer zu begrenzten Anwendung der Vorschrift in der Praxis führe. Am Beispiel der Schwierigkeiten, von einer Universität an eine andere wechseln zu können, zeigte sie den grundlegenden Reformbedarf auf. Daher befürwortete sie eine Überarbeitung der Vorschrift dahingehend, Harmonisierung im Bereich Schul- und Bildungspolitik zukünftig nicht mehr auszuschließen sondern vielmehr eine „Harmonisierung durch den Haupteingang" zuzulassen.

\section{Zur Quadratur des Kreises zwischen politischen Notwendigkeiten und verfassungsrechtlichen Limitierungen}

Im abschließenden Panel äußerte Iris Canor zunächst Zweifel am Ansatz, dass die Europäische Union mehr Harmonisierung benötige. Sie wies darauf hin, dass es stets mehrere Blickwinkel auf Sachverhalte gebe. Inwieweit nationale und konstitutionelle Identität gleichgesetzt werden könnten, müsse am Maßstab der Souveränität eines Staates festgemacht werden. Schlussendlich gab sie zu bedenken, ob nicht auch die Union seit dem Inkrafttreten des Vertrags von Lissabon eine eigene konstitutionelle Identität haben könne.

Oliver Diggelmann führte - aus der Außenperspektive der Schweiz - an, dass hinsichtlich einer ,immer engeren Union“ die demokratische Legitimierung zu oft noch nicht ernst genommen werde. Zudem sei der institutionelle Aufbau der Union zu komplex. Schließlich regte er einen vorsichtigeren Gebrauch von Metaphern wie der der Säulen oder des sich bewegenden Fahrrads an, wolle man den Bürgern das Verständnis für die Funktionsweisen der Union erleichtern.

Waltraud Hakenberg hielt zunächst fest, dass der Europäische Gerichtshof nicht wirklich eine politische Institution sei, und hob die Notwendigkeit eines solchen Gerichtshofes einerseits, der mitgliedstaatlichen Verfassungsgerichte andererseits, hervor. Sie befürwortete eine stärkere Kooperation zwischen ihnen, aber auch innerhalb des EU-Organgefüges. Die teilweise diesbezügliche Inaktivität kritisierte sie deutlich.

Jo Leinen erklärte, dass die absolute Mehrheit der EU-Maßnahmen - 99 Prozent - innerhalb der Kompetenzen erfolgt sei. Er kritisierte die jüngste Europarede des britischen Premierminister David Cameron als irreführend und gefährlich, da sie Desintegration befürworte. Er trat für „mehr Europa“ ein, welches unter anderem eine Wirtschaftsregierung beinhalten solle. Aufgrund der engen Verbundenheit zueinander sei dies notwendig. Dies erfordere freilich eine Vertragsreform. Verfassungsrechtliche Integrationslimits lehnte Leinen ab.

Siegfried Magiera, welcher das Panel leitete, begrüßte schließlich die durch den Vertrag von Lissabon eingeführte Austrittsmöglichkeit, ${ }^{16}$ welche den Staaten eine große Freiheit hinsichtlich ihres Verhältnisses zur Europäischen

16 Art. 50 EUV. 
Union ermögliche. Eine von ihr ausgehende Gefahr für die Integration mochte er nicht erkennen. Integrationslimits wollte er nur in Verfahrensvorschriften sehen, welche durch Konsensus festgelegt sein sollten. Hierbei hob er besonders die Menschenwürde, Demokratie und die Rechtsstaatlichkeit hervor.

Die abschließende Diskussionsrunde, an welcher sich alle Anwesenden beteiligten, befasste sich in erster Linie mit möglichen Vertragsänderungen und ihren Neuerungen. Umstritten war vor allem die von Jo Leinen angesprochene Möglichkeit von assoziierten Mitgliedern, welche von Christine Kaddous und Sebastian Zeitzmann abgelehnt wurde. Torsten Stein sprach sich durchaus für mehr Europa aus, dies allerdings in erster Linie zur Bewältigung der Krise. In anderen Politikbereichen sollte stattdessen der Mut bestehen, auch einmal einen Gang zurückzuschalten. Carl Otto Lenz bestritt die Notwendigkeit einer Vertragsreform, solange die gegenwärtig bestehenden Regeln nur strikt eingehalten würden. Er sah auch kein Problem mit der demokratischen Legitimation in der Europäischen Union, was von mehreren Teilnehmern in reger Diskussion anders gesehen wurde.

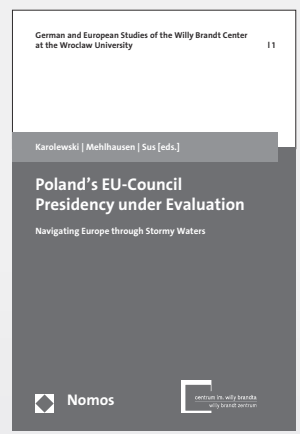

\title{
Poland's EU-Council Presidency Under Evaluation
}

\author{
Navigating Europe Through Stormy Waters \\ Herausgegeben von Ireneusz Pawel Karolew- \\ ski, Thomas Mehlhausen und Monika Sus \\ 2014, ca. 180 S., brosch., 34, $-€$ \\ ISBN 978-3-8487-0305-0 \\ (German and European Studies of the Willy \\ Brandt Center at the Wroclaw University, Bd. 1) \\ Erscheint ca. Januar 2014
}

Der Band untersucht die polnische EU-Ratspräsidentschaft im Rahmen der Änderungen durch den Vertrag von Lissabon. Er umfasst Themen wie die Östliche Partnerschaft, Energie- und Klimapolitik, die Gemeinsame Außenund Sicherheitspolitik, den EU-Erweiterungsprozess und die Aushandlung des mehrjährigen Finanzrahmens 2014-2020.

Die Autoren sind polnische und deutsche Experten.

Bestellen Sie jetzt telefonisch unter 07221/2104-37 Portofreie Buch-Bestellungen unter www.nomos-shop.de/20594

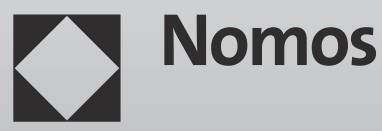

